\title{
Rechtswissenschaft tut not!
}

\section{Anmerkungen zur Tagung der Deutschen Sektion der Internationalen Vereinigung für Rechts- und Sozialphilosophie (IVR) vom 27. bis 29. September 2012 in Münster („Evolution - Entwicklung - Epigenesis des Rechts“)}

Wussten Sie schon, dass bei IBM in der Rechtsabteilung fast nur noch Nichtjuristen arbeiten? Weil Juristen zu viele Bedenken haben, etwa bei Kündigungen? Oder bei dem, was der Kündigung meist vorausgeht, nämlich der permanenten gegenseitigen Evaluation der Mitarbeiter im Netz - die dazu führt, dass nur noch die Angepassten überleben? Nun, das sind Dinge, die man auf einer Tagung eben auch erfährt, allerdings eher am Rande, in der Kaffeepause.

Die Tagung der Deutschen Sektion der Internationalen Vereinigung für Rechts- und Sozialphilosophie (IVR) fand dieses Jahr in Münster statt, und der Titel lautete: Evolution - Entwicklung - Epigenesis des Rechts. Zur Erläuterung: Epigenesis ist, was geschieht, wenn sich die Entwicklung oder Evolution ihrer selbst bewusst, wenn sie reflexiv wird. So jedenfalls die Definition der Organisatoren der Tagung, Thomas Gutmann (Zivilrecht) und Fabian Wittreck (Öffentliches Recht) sowie Bernhard Jakl (Zivilrecht) und Michael Städtler (Philosophie).

Und was geschieht bei dieser Bewusstwerdung? Nun, es entsteht u.a. die Frage, ob es im Recht so etwas wie Fortschritt gibt. Und da war die klare Ansage von Massimo La Torre (Catanzaro/Hull): Recht ist ein Fortschritt - gegenüber der bloßen Macht, gegenüber dem „Recht“ des Stärkeren, das eigentlich Nicht-Recht ist, nicht Recht, wie wir es verstehen. Denn wir verstehen unter einem „Recht“ nicht schon die faktisch-subjektive Macht, etwas durchzusetzen, sondern erst einen Anspruch, der sich aus objektivem Recht ergibt, aus einer Rechtsordnung, die ihrerseits den Anspruch auf Richtigkeit und Gerechtigkeit erhebt. Erst aus diesem objektiven Recht können sich subjektive Rechte im eigentlichen Sinn „entwickeln“ - so La Torre, der allerdings dafür plädierte, dabei auch die („,subjektive“) Perspektive der Anwälte und der Rechtsunterworfenen ernst zu nehmen. Hätte man daher nicht gut von einer CoEvolution des objektiven Rechts und der subjektiven Rechte sprechen können? La Torre hingegen entpuppte sich in der Diskussion zu seinem Vortrag („Rechtsevolution und der Anspruch auf Fortschritt") als Gegner des Evolutionismus, und Kurt Bayertz (Münster) brachte dies in der Diskussion auf die Formel: Soweit es im Recht Fortschritt gibt, ist es keine Evolution.

Klaus Günther (Frankfurt am Main) war, im Eröffnungsvortrag („Recht und Evolution“), wohl anderer Meinung gewesen. Zwar gab er mit Marx und Nietzsche der Kritik am Projekt der Moderne, an der Legitimität der Neuzeit (Blumenberg), viel Raum: Kritik an ihrem Glauben an Fortschritt durch Recht. Aber Kritik am Recht als bloß verbrämter Macht ist selbst durchdrungen von Macht (Foucault), ist eine 
Machttechnik. Und so entpuppte Günther sich am Ende als jemand, der auf die Kritische Theorie und ihren Gedanken etwa eines Fortschritts im moralischen Bewusstsein (Kohlberg, Habermas) mit einer gewissen Distanz zurückblickte. Stattdessen hielt er im Geiste der Systemtheorie (Luhmann) Kommunikationen für eigenständige Entitäten (Fögen) und damit der Evolution zugänglich. Im Einzelnen: Evolution der Kooperation (Tomasello), indem geteilte Intentionen möglich werden; Evolution von Normen und Institutionen (Henrich, Boyd, Ostrom), zum Beispiel durch „altruistisches Bestrafen “ unkooperativen Verhaltens durch die Gruppenmitglieder selbst (s.o. IBM!); schließlich etwas, das Günther „Evolution des Raumes der Gründe“ nannte - denn Normen sind intern zugleich Gründe (Raz), und das Recht verstärkt sie extern durch Kalibrierung, intern durch Institutionalisierung.

Ist also Recht nur eine Stabilisierung von etwas, das schon vor dem Recht da ist? Bei Günther kam das Recht, wie wir es kennen, in der Tat vor als etwas, das sich „erst auf der Stufe der funktionalen Differenzierung“ der Gesellschaft „als Einheit“ erleben darf. Aber das verpasst doch die Pointe! Denn die Bedeutung des Rechts für die Entwicklung der modernen Gesellschaft ist: Das Recht war das erste Funktionssystem der Gesellschaft! Erst als man in Bologna im 11. Jahrhundert mit einem neuen Big Book gegen die Bibel argumentieren konnte, wurde es möglich, Recht von „Moral“ und Gottes Geboten zu trennen und es als ein eigenes, „autopoietisches“ System von Kommunikationen zu entfalten. Nach diesem Vorbild zerfaserte dann später die Gesellschaft in die vielen weiteren Funktionssysteme (Wirtschaft, Politik, Wissenschaft, Kunst, Religion usw.), die allesamt nach ihren eigenen Regeln leben.

Aber wie soll es mit diesen Funktionssystemen weitergehen? Nun, auch sie sind dem Wandel, der Entwicklung unterworfen. Sie können sogar, als historische Errungenschaft, wieder verlorengehen - und mit ihnen die Freiheit, die sie ermöglichen. Deshalb darf man auch nicht darauf vertrauen, dass sich für das Recht, wie wir es verstehen, alles von selbst zum Besten „entwickeln“ wird. Vielmehr wird das Recht, gerade um der (modernen) Gesellschaft willen, seinen Eigenwert verteidigen müssen. Bedeutet dies nun fiat iustitia, et pereat mundus - es geschehe Gerechtigkeit, mag auch die Welt darüber untergehen? Nein: fiat ius, ne pereat societas - man produziere Recht, damit die Gesellschaft nicht verdirbt, d.h. völlig auseinanderfällt.

Aber wie soll das gelingen?

Es gehört zu den Eigenheiten von IVR-Tagungen, dass man am Nachmittag, nach den beiden Plenarvorträgen des Vormittags, immer unzufrieden ist. Denn man verpasst notwendigerweise eine der beiden Sektionen, in die sich das Programm aufspaltet - am Freitag war es entweder die öffentlich-rechtliche oder die privatrechtli- 
che. Ich habe die privatrechtliche gewählt, und dort ging es genau um die soeben gestellte Frage nach dem Überleben des Rechts in einer Gesellschaft, die sich rasant entwickelt - ohne dass man wissen kann wohin.

Wenn man den ersten Vortrag nimmt, wurde zumindest eines klar: Auf einen wichtigen Bundesgenossen sollte sich das Recht nicht mehr verlassen, nämlich auf den Staat. Das zeigte Dan Wielsch (Köln) unter dem Titel „Vergesellschaftung von Recht" am Beispiel des Eigentums, speziell am Geistigen Eigentum, noch spezieller an den Intellectual Property Rights im Internet, ganz speziell an Zugangsregeln. These: Da der Staat hier an die Grenzen seiner Macht stoße, müsse eben „die Gesellschaft" selbst die nötigen Regeln schaffen. Die Gesellschaft? Wer ist das? Auf Nachfrage: Die „Gruppen von Produktiven“ schaffen sich ihre Regeln selbst. Oder wie Wielsch schon vorher formuliert hatte: Es gehe um eine Neubestimmung politischer (sic!) Autonomie jenseits staatlicher Rechtsetzungsverfahren, um die Ausübung privater Autonomie zur Erhaltung ihrer Voraussetzungen. All dies übrigens mit praktischer Erfahrung gesättigt und systemtheoretisch unterlegt.

Und doch bleibt Skepsis. Denn wenn der Staat, das Zentrum des Funktionssystems Politik, wegfällt, wer ist dann die Autorität, die das Recht schafft (Hobbes)? Wer ist es, der dann die Unparteilichkeit des (objektiven) Rechts gewährleistet - zumal nach Wielsch vor allem sie es ist, die das Recht rechtfertigt? Etwa der Markt (es geht ja um Eigentumsrechte)? Wohl kaum, denn, frei nach Böckenförde: Auch der Markt lebt von Voraussetzungen, die er selbst nicht garantieren kann. Und die Banken, das Zentrum des Funktionssystems Wirtschaft, garantieren dies nach allen jüngsten Erfahrungen erst recht nicht.

Im Gegenteil: Sie, die Banken, waren auf „Stütze“, auf Sozialhilfe von Seiten des Staates angewiesen, und zwar auf Kosten des Rechts. Dies war das Thema von Marc Amstutz“ (Fribourg) Vortrag über „Grenzverwischungen: Zum Konzept einer evolutionären Regulierung der Finanzmarktkrise“. Amstutz, der Schweizer, machte zuerst ganz klar, dass die Finanzkrise keine Wirtschaftskrise, sondern eine Gesellschaftskrise gewesen sein (jawohl!). Und sie entstand und entwickelte sich dadurch, dass Systemgrenzen aufgelöst oder verwischt wurden - hier die Grenzen der Funktionssysteme Wirtschaft, Politik und Recht. Und wodurch wurden sie verwischt? Indem der Code, nach dem diese Systeme funktionieren, aufgeweicht wurde: Es ging eben nicht mehr nur - binär - um zahlen/nicht-zahlen (Wirtschaft), um Regierung/ Opposition (Politik), um Recht/Unrecht (Recht). Sondern es wurde allen Kommunikationen, die sich an diesen Codes orientierten, etwas Drittes beigemischt: Rücksicht auf die Umwelt, die kollabieren könnte, wenn man es mit dem Code zu weit triebe (s.o.: fiat iustitia ...). Und dies, möchte man ergänzen, gewiss aus Angst davor, mit in den Strudel gerissen zu werden. Wie auch immer: Das Fallbeispiel, das Amstutz vorexerzierte, war die Schweizer Bank UBS, die den Auflagen der US-Be- 
hörden nur dann hätte genügen können, wenn sie ihren Kunden gegenüber das Bankgeheimnis verletzt hätte. Und wenn sie den US-Auflagen nicht genügte, drohte ihr, wegen enormer Sanktionen, der Konkurs. In dieser Zwickmühle suchte die UBS als Nothelfer den Staat, und die Schweizer Behörden ließen sich wirklich den Schwarzen Peter zuschieben: Sie wiesen die Bank an, die Daten unter Verstoß gegen das Bankgeheimnis herauszugeben. Und das Recht?

Nun, das Recht war das System, das sich am meisten demütigen lassen musste. Zwar hob das Bundesverwaltungsgericht der Schweiz den Befehl der Behörde auf. Aber dieser Entscheid wurde seinerseits vom Bundesgericht aufgehoben, mit der bemerkenswerten Begründung: Zwar sei eines der Tatbestandsmerkmale des Notrechts aus Art. 185 Bundesverfassung, auf das die Maßnahme gestützt wurde, nicht erfüllt. Dennoch hätten Bundesrat (Regierung) und FINMA (die Behörde) die Notklausel gemeinsam ausüben dürfen - letztlich aus rein politischen Gründen. Damit war das Recht eingeknickt vor der Politik wie zuvor die Politik vor der Wirtschaft.

Was tun, um so etwas künftig zu verhindern - und damit die Systemgrenzen zu stärken? Nun, vielleicht ist es naiv zu sagen: Man muss trotz allem auf dem Code beharren und zu Banken und Regierungen, die in der Krise sind (oder dies vorgeben), angesichts des Rechts sagen: „Das interessiert uns nicht. Wir kennen nur Recht/Unrecht." Amstutz suchte die Lösung nicht auf der Ebene des Codes, sondern, wie Luhmann sagen würde, auf der Ebene der Programme, d.h. er fragte: Welche Rechtsregeln könnten für alle Akteure so sinnvoll und überzeugend sein, dass sie nicht erneut in Versuchung geraten, ihre Probleme wie Parasiten (sic!) von anderen Systemen lösen zu lassen? Und schlug als mögliches Modell im Anschluss an Walsh eine „institution-based financial regulation" vor - ein Unternehmensrecht, das zwingende institutionelle Vorgaben enthält, aber den Firmen genug Raum lässt, „mit der sozialen Evolution Schritt zu halten“.

Ich bleibe skeptisch. Kann man wirklich die Grenzen des Rechts, d.h. seinen Code, stärken, indem man sich bemüht, die Probleme anderer Systeme, hier der Wirtschaft, zu lösen? Anders gewendet: Was nützt es dem Recht, vernünftig zu sein, wenn es ihm an der Kraft fehlt, sich selbst zu behaupten? (Man denke auch an die Heuchelei, mit der das Bundesverfassungsgericht bei der Bundestagsauflösung 1982 eine politische Entscheidung als rechtliche verpackt hat.) Noch anders formuliert: Sollte das Recht für die Rechtsunterworfenen attraktiv sein, oder sollte es sich auf eine Macht stützen, die es durchsetzt?

$* * *$

Man wird dies vielleicht für die verschiedenen Rechtsgebiete unterschiedlich beantworten müssen. Im Privatrecht war die Tendenz jedenfalls klar: Recht sollte attraktiv 
sein. Dies unterstrich - um zeitlich vorzugreifen - noch einmal der zweite Plenarredner des zweiten Tages: Nils Jansen (Münster) über „Informelle Autoritäten in der Privatrechtsentwicklung“. Gemeint waren, praktisch gesehen, nicht-legislative Kodifikationen im transnationalen Privatrecht, konkret UNIDROIT (Principles of International Commercial Contracts) und PECL (European Principles of Contract Law). Theoretisch gesehen setzte sich Jansen zunächst mit dem Gegensatz von auctoritas und potestas auseinander, sodann hinsichtlich der Autorität mit ihrem Verhältnis zur Anerkennung. Verblüffend dann die praktische Beobachtung: Was faktisch wie eine Norm auftritt - hier UNIDROIT, zu denen es Kommentare gibt, die eben genau wie der „Palandt“ oder der „Maunz/Dürig“ aussehen -, wird auch als Norm akzeptiert. Jansen meinte deshalb, theoretisch, der alte Begriff der Geltung passe nicht mehr. Aber das lag vielleicht an seiner Sozialisation unter Öffentlichrechtlern?

Auf Seiten der Öffentlichrechtler gab es jedenfalls, wie die Diskussion zeigte, zum Teil blankes Entsetzen: Hier maße sich ein informelles Regelwerk Autorität an, die ihm nicht zustehe. Und in der Tat: Auf den ersten Blick scheint es so, als werde das rigide binäre Schema der Geltung („gilt/gilt nicht“) durch das analoge Schema der Anerkennung (,mehr oder weniger“) ersetzt. Aber auf den zweiten Blick muss man gerade auch mit Kelsen sagen: Hinter der Geltung steht nicht, wie die Deutschen immer denken, der Staat, sondern die Grundnorm. Die Grundnorm aber ist eine (nur) gedankliche Voraussetzung, die wir machen müssen, um so etwas wie Recht zu erklären, und Kelsen hat am Beispiel des Völkerrechts selbst gezeigt, dass die Grundnorm auf bloßer Anerkennung fußen kann. Daher muss für Jansens Thema wohl gelten: Wer sich UNIDROIT unterwirft, für den gilt es eben. Und wenn die Unterwerfung auf einer Fehlvorstellung über den Autor beruht, dann ist das ein unbeachtlicher Motivirrtum.

Aber die Fehlvorstellung über den Autor des Rechts (oder „Rechts“?) ist, als solche, interessant. Worauf beruht sie? Nun, dies führt zum ersten Plenarvortrag des zweiten Tages, zu Thomas Vesting (Frankfurt am Main) mit seinem Referat über die „Mediengeschichte des Rechts“. Dieser Vortrag war historisch natürlich weit gespannt von den Kulturen mündlicher Rechtstradition über die Bücher des Rechts und die Massenmedien des Rechts bis zu Netzwerken des Rechts, in denen sich der Unterschied zwischen Markt und Organisation auflöst. Vesting setzte sich zudem intensiv mit der Auflösung der Einheit des klassischen Subjekts zugunsten einer Umstellung auf Evolutionstheorie auseinander, mit besonderem Akzent auf der Rolle der Kultur, und es gab auch Kritik an der Fixierung sogar Luhmanns auf das Römische Recht zu Lasten der griechischen und jüdischen Tradition. Am Ende blieb die Erkenntnis, dass wir, als die zerrissenen Subjekte, die wir heute sind, nicht mehr von vornherein den Gehalt unserer wechselseitigen Akzeptanz kennen können. „Convention as a 
whole is now looked upon not as a firm inheritance from the past but a continuing improvisation in the face of problems we no longer understand" (Cavell).

Also der Vorhang zu und alle Fragen offen (Brecht)?

Nun, vielleicht kann man sagen: Zeig mir die Gesellschaft, in der du lebst, und ich sage dir die Rechtstheorie, die du hast. Und da sehen wir, natürlich, dass die (Welt-)Gesellschaft, in der wir leben, uns nicht mehr viel an Einheit und Ganzheit („a whole“) bietet. Sie verlangt uns in der Tat einiges an Improvisation ab (Cavell ist ursprünglich Musiker). Wie soll sich in dieser Welt das Recht weiterentwickeln?

Nun, es wird versuchen müssen, in irgendeiner Weise seine Einheit und Ganzheit zu bewahren, zu verteidigen, vielleicht sogar: wiederzuerlangen (immerhin endet Luhmanns „Recht der Gesellschaft“ mit einem Eventual-Abgesang auf die abendländische Kultur, die, geprägt vom Recht, welthistorisch nur eine Episode gewesen sein könnte). Aber wie soll das gelingen?

Nun, das Recht wird seine Einheit und Ganzheit wohl nicht bewahren können, wenn es lediglich in Einzelbereichen „vernünftige Lösungen“ anbietet (so die Tendenz der Privatrechtler). Es muss vielmehr weiterhin eine Welt für sich bleiben, und dafür braucht es Verbündete, die das Recht dort stützen, wo es nicht aus Überzeugung befolgt wird, sei es im Strafrecht und im sonstigen Öffentlichen Recht, aber auch selbstverständlich - im innerstaatlichen Zivilrecht. (Interessanterweise hatten fast alle privatrechtlichen Vorträge der Tagung einen internationalen Bezug; allein Lorenz Kähler (Bremen), als Dritter der Sektion am Freitag, blieb bei seinem Thema „Reservegrundsätze in der Entwicklung des Privatrechts“ in der Welt der obersten deutschen Gerichte.)

Aber wie soll das Recht sich behaupten, wenn der Staat, wie mehrfach angeklungen, als Verbündeter wegfällt oder gar vor der Wirtschaft in die Knie geht?

Nun, es bleibt dann wohl nur: die Wissenschaft. Und so ist ja auch eigentlich alles entstanden: In Bologna war die Rechtsschule der Keim dessen, was wir „Universität“ nennen, und universitas heißt eben „Gesamtheit“ der Wissenschaften. Ohne (Rechts-)Wissenschaft würde es so etwas wie UNIDROIT samt dazugehörigen Kommentaren nicht geben, genauer: nicht ohne eine Wissenschaft, die ebenso wie das Recht (Wielsch) die entscheidende Legitimation aus ihrer Unparteilichkeit gewinnt. Und deshalb sollten wir vielleicht dafür kämpfen, dass die Universitäten das Zentrum der Wissenschaft bleiben, dass, mit anderen Worten, die Forschung nicht in SonderInstitute verstreut wird. Nur die Universität bewahrt mit ihrer Einheit der Wissenschaften die Objektivität aller Wissenschaften - auch der Rechtswissenschaft. Nur 
in ihr gelingt zudem die strukturelle Kopplung von Wissenschaft und Gesamtgesellschaft: in Gestalt des Studenten (als Institution).

Es bleibt nachzutragen, worüber hier nicht oder nicht vertieft berichtet werden kann. Am Begrüßungsabend (Donnerstag) warb der Fachphilosoph Michael Quante (Münster), Präsident der Deutschen Gesellschaft für Philosophie, für eine stärkere Kooperation von juristenbetriebener "Rechtsphilosophie“ und fachphilosophisch betriebener „Philosophie des Rechts“. Das ist sehr zu begrüßen, aus meiner Sicht wäre allerdings hinzufügen: Kooperation nicht nur in Sonderforschungsbereichen (während man als Juraprofessor im Übrigen vom ersten Semester an Examensvorbereitung nach Repetitorenart betreibt).

In der Sektion Öffentliches Recht (Freitagnachmittag) sprach Martin Schulte (Dresden) über „Evolution des Rechts - dargestellt am Beispiel des Verhältnisses von staatlichem und kirchlichem Recht“, gefolgt von Stefan Oeter (Hamburg) zum Thema „Entwicklungsperspektiven im Völkerrecht" und von Andreas Funke (Erlangen) mit dem Vortrag „Denken in Entwicklungen - Gewissheitsverluste durch Europäisierung und Internationalisierung? “ Zu allem kann ich nur vom Hörensagen berichten: Es war gut.

Die Sektion Philosophie (Samstagnachmittag) bestritten in der Tat Fachphilosophen, zunächst Günter Zöller (München), der unter dem Titel „Genese und Geltung. Der Entwicklungsgedanke in der Rechtsreflexion von Kant, Fichte und Hegel“ namentlich über den Begriff „Epigenesis“ aufklärte. Oliver Eberl (Darmstadt/Hannover) beschäftigte sich mit dem Topos „Überwindung der ,Barbarei‘ als Grund der Rechtsentwicklung “ - und zog dabei die Entwicklungslinie vom Begriff des Barbaren (Antike bis Renaissance) über das Konstrukt „Naturzustand“ bei Hobbes bis zu dessen Überwindung bei Kant. Dahinter stand, auch wenn dies nicht eindeutig gesagt wurde, offensichtlich ein Bekenntnis zum Fortschritt: zum Fortschritt durch Recht. Denn erst das Recht ermöglicht es, sich ,auf zivile Art“ zu streiten (Kant) - und eben nicht barbarisch.

Zur Sektion Strafrecht (Samstagnachmittag) zunächst nur vom Hörensagen: Kai Ambos (Göttingen) sprach „Zum Ius puniendi des Völkerstrafrechts“, Beatrice Brunhöber (Berlin) über „Rechtsgutslehre und Konstitutionalisierung des Strafrechts" - beide sehr anregend. Ein Bericht aus eigener Anschauung ist möglich über Benno Zabel (Leipzig) und seinen Vortrag „Das Wissen im Subjekt. Zur Bedeutung des inneren Forums im Wandel strafrechtlicher Sozialkontrolle“. Dahinter verbarg sich zunächst eine einfühlsame Auseinandersetzung mit der „Gewissenserforschung “ durch Folter auf Grundlage der Consitutio Criminalis Carolina (1532) und in den 
Hexen- und Ketzerprozessen. Hinter dieser Gewissenserforschung stand, wie Zabel darlegte, die ungeheure Angst, sich Gottes Zorn zuzuziehen: sei es, weil man einen Sünder ungestraft ließ, sei es, weil man den Falschen verurteilte. Erst als man daher mangels Schutz durch Gott auf Sicherheit durch den Staat baute, wurde freie Beweiswürdigung möglich. Dennoch wollte Zabel die Rechtsgeschichte nicht als Fortschrittsgeschichte lesen („Strafrecht muss Ängste abbauen; kann es aber nicht“). Eberl hatte immerhin formuliert, dass es Rückfälle geben könne: in die Barbarei. Und dass man dies immer nur bei den Anderen wahrnimmt, nicht bei sich selbst, dies hätten für unsere abendländisch durchsonnte Kultur am besten die Hexenprozesse gezeigt.

$* * *$

Evolution, Entwicklung, Epigenesis des Rechts: Was geschieht, wenn das Recht sich dessen bewusst wird, dass - vielleicht sogar: wie - es sich entwickelt? Nun, dann muss das Recht wohl, zwangsläufig, philosophisch werden, „und das heißt vordergründig schlicht: ganzheitliches Fragen, Fragen nach dem Grund, auf dem man sich bewegt" (Hollerbach). Die Tagung der IVR hat vielfach gezeigt: Der Grund, auf dem sich das Recht bewegt, scheint schwankend geworden zu sein. Aber vielleicht ist er immer schon schwankend gewesen? Vielleicht hatten wir nur das Glück, eine so lange Zeit des Friedens zu erleben? Natürlich fiel in Münster auch der Satz: pax optima rerum, der Frieden ist das beste aller Dinge. Dass dieser Frieden ohne Recht nicht zu haben ist, daran muss die Menschheit wohl immer wieder erinnert werden, sei es im Großen oder auch, wie bei IBM, im Kleinen.

Joachim Lege, Greifswald 